\title{
Multiphonon anharmonic decay of a quantum mode
}

\author{
V.Hizhnyakov \\ Institute of Theoretical Physics, University of Tartu, Tähe 4, 51014 Tartu, Estonia \\ Institute of Physics, University of Tartu, Riia 142, 51014 Tartu, Estonia
}

\begin{abstract}
A nonperturbative theory of multiphonon anharmonic transitions between energy levels of a local mode is presented. It is shown that the rate of transitions rearranges near the critical level number $n_{c r}$ : at smaller $n$ the process slows down, while at larger $n$ it accelerates in time, causing a jump-like loss of energy followed by the generation of phonon bursts. Depending on parameters, phonons are emitted in pairs, triplets etc.
\end{abstract}

PACS: 71.23.A, 71.55.-i

The nonlinear dynamics of strong vibrational excitations in crystals has attracted remarkable attention during the last years. The opportunities of applying novel numerical techniques play an essential role in the investigation of nonlinear discrete systems, allowing for the observation of different phenomena. One of the effects, the existence of localized vibrations in a pure anharmonic lattice (so-called intrinsic local modes (ILM) or self-localized solitons (SLS) 15 $_{5}$, is of particular interest, as it links local lattice dynamics to the physics of solitons and underlines, in general, the importance of strongly excited local modes. So far the research of strong anharmonic effects in lattice dynamics as well as molecular dynamics simulations of a nonlinear lattice have been mainly carried out within the framework of classical mechanics. However, quantum effects are important here as they lead to extra mechanisms in the decay of vibrational excitations. This holds especially for the modes with frequencies above the phonon spectrum because they, being singly excited, do not decay at all in the classical limit - it is because all their harmonics are out of resonance with the linear spectrum which makes it impossible to transfer energy to phonons $\bar{E}$. Quantum decay of the ILMs has been discussed by Ovchinnikov 1 in the framework of standard perturbation theory. However, this theory may not be applicable here because ILMs exist only when their amplitudes (energy) are large enough; therefore, one cannot assume that anharmonic interactions are weak. To understand how relaxation proceeds when the energy of the mode is large, one should develop a nonperturbative theory of multiphonon transitions between the quantum levels due to anharmpnic interaction with the phonon continuum.

Recently, int 8 we proposed a theory of anharmonic damping of a strongly excited mode, supposing that the latter can be considered classically (phonons in this theory are described quantum-mechanically). The idea of Ref. 6 . to consider the effect of the strongly excited mode upon the phonon operators. The goal was to find a transformation of these operators in time, and, from that, to obtain the probability of creation of a phonon. Adding all the created phonons allows one to find the energy loss in the mode.

The realization of the method in the case of quadratic interaction of the mode with respect to phonon coordinate operators $\hat{x}_{i}$ is straightforward: the phonon Hamiltonian with the anharmonic interaction $\sum_{i i^{\prime}} V_{l i i^{\prime}}\left(x_{l}(t)\right) \hat{x}_{i} \hat{x}_{i^{\prime}}$, where $x_{l}(t)=A \cos \left(\omega_{l} t\right)$ is a classical time-dependent coordinate of the local mode, can be diagonalized exactly for an arbitrary amplitude $A$ of the mode. This is achieved by the transformation $\hat{b}_{j}(t)=\sum_{i}\left[\mu_{i j}(t) \hat{a}_{i}+\nu_{i j}(t) \hat{a}_{i}^{+}\right]$where $\hat{a}_{i}$ and $\hat{a}_{i^{\prime}}^{+}$are initial destruction and creation operators of phonons (for explicit expressions for $\mu_{i j}$ and $\nu_{i j}$, seed). The initial zero-point state, $|0\rangle$, is not the zeroth state of the operators $\hat{b}_{j}$. This means that phonons are generated in a lattice. The number of created phonons $j$ equals $n_{j}=\sum_{i}\left|\nu_{i j}\right|^{2}$. For times $t \gg \omega_{i}^{-1}$ the transformation simplifies 8 : $\mu_{i j}, \nu_{i j} \sim \delta_{i j}$ and the rate of energy relaxation becomes

$$
-\dot{E}_{l} \simeq \sum_{i} \hbar \omega_{i} d\left|\nu_{i}\right|^{2} / d t
$$

Int, it was found that for a large energy mode, the relaxation rate diminishes with energy: $-\dot{E}_{l} \sim A^{-2}$, i.e. relaxation accelerates with time. This result contradicts standard perturbation theory. Yet, the interpretation of this surprising result is straightforward - the decay is of purely quantum mechanical origin and, therefore, it should disappear in the classical limit $E_{l} \rightarrow \infty$. It was also shown that the acceleration of the process continues down to an energy $E_{c r}$ (which typically corresponds to $E_{l} \sim 10-30$ vibrational quanta, or $A \sim 0.2 \AA-0.5 \AA$ ) when the rate becomes high (of the order of $\hbar \omega_{l}^{2}$ ) and a sharp relaxation jump takes place accompanied by generation of a burst of phonons. The latter are emitted in pairs. After the first jump, several other jumps may occur. For small energies relaxation slows down exponentially in agreement with perturbation theory. 
The theory of Ref $\mathrm{A}$. $\mathrm{B}$ considers the relaxation of the mode as a continuous process where the energy of a classical subsystem is diminishing. In this communication, the effects of the quantum origin of the states of the mode and of the transitions between its levels will be considered. Here we restrict ourselves to quadratic terms in the expansion of the potential energy with respect to phonon operators: $\hat{U}=U_{0}\left(\hat{x}_{l}\right)+\sum_{i} U_{1 i}\left(\hat{x}_{l}\right) \hat{x}_{i}+\sum_{i i^{\prime}} U_{2 i i^{\prime}}\left(\hat{x}_{l}\right) \hat{x}_{i} \hat{x}_{i^{\prime}} / 2$; the potential of the local mode, $U_{0}\left(\hat{x}_{l}\right)$, is supposed to be anharmonic, and so are the factors $U_{1 i}\left(\hat{x}_{l}\right)$ and $U_{2 i i^{\prime}}\left(\hat{x}_{l}\right)$ : they depend on the displacement operator $\hat{x}_{l}$ due to higher order anharmonicities. To clarify the range of the applicability of the approximation, we observe that all anharmonic terms become comparable to each other for shifts of an atomic distance of typically $1 \AA$ 道. E.g. the harmonic $V_{l}^{(2)} x_{l}^{2}$ and anharmonic $\left(V_{l}^{(3)} / 3+V_{l}^{(4)} x_{l} / 12\right) x_{l}^{3}$ terms in the expansion of $U_{0}\left(x_{l}\right)$ are of the same order of magnitude for $x_{l} \sim 1 \AA$, while the terms $V_{i i^{\prime}}^{(2)} x_{i} x_{i^{\prime}}$ and $\left(V_{l i i^{\prime}}^{(3)}+V_{l i i^{\prime}}^{(4)} x_{l} / 2\right) x_{l} x_{i} x_{i^{\prime}}$ in the expansion of $U_{2 i i^{\prime}}\left(x_{l}\right) x_{i} x i^{\prime}$ are of the same order of magnitude for $x_{l} \sim 0.3 \AA$. Below we take into account these terms while we consider just local modes with the amplitude $A \sim 0.3 \AA$ or less. For such amplitudes the terms $\sim x_{i} x_{i^{\prime}} x_{i}$ " are already much smaller and are neglected.

Within this approximation, the interaction of the local mode with phonons has the form

$$
\hat{H}_{i n t}=\sum_{i} V_{l l i}(\hat{n}) \hat{x}_{i}+\frac{1}{2} \sum_{i i^{\prime}}\left[V_{l i i^{\prime}}(\hat{n}) \hat{a}_{l}+H . c .\right] \hat{x}_{i} \hat{x}_{i^{\prime}},
$$

where $\hat{n}=\hat{a}_{l}^{+} \hat{a}_{l}, \hat{a}_{l}^{+}$and $\hat{a}_{l}$ are the creation and destruction operators; terms $\sim \hat{a}_{l}^{+{ }^{k}}$ as well as $\sim \hat{a}_{l}^{k}, k=2,3, \ldots$ are neglected; they describe transitions between the levels $n$ and $n-k$ and could be considered analogously. Below the operators $V_{l l i}(\hat{n})$ and $V_{l i i^{\prime}}(\hat{n})$ stemming from $U_{1 i}$ and $U_{2 i i^{\prime}}$ respectively, are applied only to the initial number state $|n\rangle$ of the local mode. Therefore the $V(\hat{n})$-operators can be replaced by $C$-functions of $n$. In this approximation, using shifted phonon operators $\hat{x}_{i}^{\prime}=\hat{x}_{i}+x_{0 i}$, with $x_{0 i}=V_{l l i} \omega_{i}^{-2}$, the interaction part of the hamiltonian reads

$$
\hat{H}_{i n t}^{\prime}=\sum_{i} \hat{V}_{l i} \hat{x}_{i}^{\prime}+\frac{1}{2} \sum_{i i^{\prime}} V_{l i i^{\prime}}\left(\hat{a}_{l}^{+}+\hat{a}_{l}\right) \hat{x}_{i}^{\prime} \hat{x}_{i^{\prime}}^{\prime}
$$

where $\hat{V}_{l i}=\bar{V}_{l i}\left(\hat{a}_{l}^{+}+\hat{a}_{l}\right)+V_{l l i}(\hat{n}-n), \bar{V}_{l i}=-\sum_{i^{\prime}} V_{l i i^{\prime}} V_{l l i} \omega_{i}^{-2}($ all $V$-factors depend on $n)$.

Let us present the operators of the phonon coordinates in the form $\hat{x}_{i}^{\prime}(t)=\left(\hbar / 2 \omega_{i}\right)\left[\hat{g}_{i}(t) \hat{a}_{i}+H . c.\right]$. Here the operators $\hat{g}_{i}(t)$ account for the time dependence of $\hat{x}_{i}^{\prime}(t)$. This dependence is determined by the equations of motion

$$
\ddot{\hat{g}}_{i}+\omega_{i}^{2} \hat{g}_{i}=-\omega_{i}\left(\hat{V}_{l i}+\sum_{i^{\prime}} V_{l i i^{\prime}} \hat{g}_{i^{\prime}}\left(\hat{a}_{l}+\hat{a}_{l}^{+}\right)\right)
$$

and by the initial conditions $\hat{g}_{i}(t)=e^{-i \omega_{i} t+i \phi_{i}}, t \rightarrow 0, \phi_{i}$ is the random phase (the final expression should be averaged over $\phi_{i}$ ). The operators $\hat{g}_{i}$ do not commute with $\hat{a}_{l}+\hat{a}_{l}^{+}$; the order of the operators in right-hand side of Eq. (3) depends on whether one considers the operators $\hat{g}_{i}$ or the operators $\hat{g}_{i}^{+}$; the opposite order describes $\hat{g}_{i}^{+}$. The integral form of the equations (3) is the following:

$$
\hat{g}_{i}(t)=e^{-i \omega_{i} t+i \phi_{i}}+\int_{0}^{t} d t^{\prime} \sin \left(\omega_{i}\left(t-t^{\prime}\right)\right)\left[\hat{V}_{l i}\left(t^{\prime}\right)+\sum_{i^{\prime}}\left(\bar{e}_{i} V_{l} \bar{e}_{i^{\prime}}\right) \hat{g}_{i^{\prime}}\left(t^{\prime}\right)\left(\hat{a}_{l}\left(t^{\prime}\right)+\hat{a}_{l}^{+}\left(t^{\prime}\right)\right)\right] .
$$

Here $\left(\bar{e}_{i} V_{l} \bar{e}_{i^{\prime}}\right) \equiv V_{l i i^{\prime}}=\sum_{m m^{\prime}} \bar{e}_{i m} V_{l m m^{\prime}} \bar{e}_{i^{\prime} m^{\prime}}, m$ is the index of Cartesian coordinates of the atoms in the lattice, $\bar{e}_{i m}=e_{i m} \omega_{i}^{-1 / 2}, e_{i m}$ are projections of the reduced displacements $x_{m}$ to the normal coordinates.

We assume that relaxation of the mode is slow as compared to its frequency. Then describing the decay of the $n$-th level caused by the $n \rightarrow n-1$ transition, one can neglect all other levels of the mode. This means that one can use the rotating wave approximation when only terms with creation and destruction operators, being arranged alternatingly in the iteration series for the $\hat{g}_{i}$, are taken into account. Let us consider in this approximation the large time asymptotics of the two-time correlation function $\left\langle n\left|\hat{x}_{i}^{\prime}(t+\tau) \hat{x}_{i}^{\prime}(t)\right| n\right\rangle$ for $t \gg \tau \sim \omega_{i}^{-1}$. Here $|n\rangle$ denotes the $n$th state of the mode and the ground state of phonons. Only the matrix elements $\left\langle n\left|\hat{g}_{i}\right| n\right\rangle$ and $\left\langle n\left|\hat{g}_{i}\right| n-1\right\rangle$ contribute to this asymptotics $\left(|n-1\rangle \equiv n^{-1 / 2} \hat{a}_{l}|n\rangle\right)$. The terms $\sim V_{l l i}(\hat{n}-n)$ of the expression for $\hat{g}_{i}(t)$ give zero; the terms $\sim \bar{V}_{l i}\left(\hat{a}_{l}+\hat{a}_{l}^{+}\right)$of this expression oscillate fast and, therefore, give small contribution which can be neglected (note, that the basic time-dependence of the operators $\hat{a}_{l}$ and $\hat{a}_{l}^{+}$is given by the factors $e^{-i \omega_{l} t}$ and $e^{i \omega_{l} t}$ respectively). In this approximation $\left\langle n\left|\hat{x}_{i}^{\prime}(t+\tau) \hat{x}_{i}^{\prime}(t)\right| n\right\rangle \approx\left(\hbar / 2 \omega_{i}\right)\left[\left|\mu_{i}(t)\right|^{2} e^{-i \omega_{i} \tau}+\left|\nu_{i}(t)\right|^{2} e^{i \omega_{i} \tau}\right], t \gg \tau \sim \omega_{i}^{-1}$, where

$$
\mu_{i}(t) \approx e^{i \phi_{i}}-(i / 2) \int_{0}^{t} d t^{\prime} \sum_{i^{\prime}}\left(\bar{e}_{i} V_{l} \bar{e}_{i^{\prime}}\right) e^{i\left(\omega_{i}+\omega_{i^{\prime}}\right) t^{\prime}} \nu_{i^{\prime}}\left(t^{\prime}\right)\left\langle n-1\left|\hat{a}_{l}\left(t^{\prime}\right)\right| n\right\rangle
$$




$$
\nu_{i}(t) \approx(i / 2) \int_{0}^{t} d t^{\prime} \sum_{i^{\prime}}\left(\bar{e}_{i} V_{l} \bar{e}_{i^{\prime}}\right) e^{-i\left(\omega_{i}+\omega_{i^{\prime}}\right) t^{\prime}} \mu_{i^{\prime}}\left(t^{\prime}\right)\left\langle n\left|\hat{a}_{l}^{+}\left(t^{\prime}\right)\right| n-1\right\rangle
$$

$\left(\mu_{i} \sim\left\langle n\left|\hat{g}_{i}\right| n\right\rangle, \nu_{i} \sim\left\langle n\left|\hat{g}_{i}\right| n-1\right\rangle\right.$; here it was taken into account that $\left.\left\langle n\left|\hat{a}_{l}^{+}\left(t^{\prime}\right)\right| n-1\right\rangle\left\langle n-1\left|\hat{a}_{l}(t)\right| n\right\rangle \cong\left\langle n\left|\hat{a}_{l}^{+}\left(t^{\prime}\right) \hat{a}_{l}(t)\right| n\right\rangle\right)$. This asymptotics corresponds to the following transformation of phonon operators : $\hat{b}_{i} \simeq \mu_{i} \hat{a}_{i}+\nu_{i}^{*} \hat{a}_{i}^{+}$. To find this transformation explicitly we present the above expression for $\nu_{i}(t)$ in the form

$$
\nu_{i}(t) \approx(i / 2) \int_{0}^{t} d t^{\prime} e^{-i \omega_{i} t^{\prime}}\left(\bar{e}_{i} V_{l} D\left(t^{\prime}\right)\right)\left\langle n\left|\hat{a}_{l}^{+}\left(t^{\prime}\right)\right| n-1\right\rangle .
$$

Here $D(t)=\sum_{i} e^{-i \omega_{i} t} \bar{e}_{i} \mu_{i}(t)$ satisfies the equation

$$
D(t)=D_{0}(t)+\int_{0}^{\infty} d t^{\prime} \int_{0}^{\infty} d t^{\prime \prime} G\left(t-t^{\prime}\right) V_{l} G^{*}\left(t^{\prime}-t^{\prime \prime}\right) V_{l} F\left(t^{\prime}-t^{\prime \prime}\right) D\left(t^{\prime \prime}\right),
$$

$D_{0}(t)=\sum_{i} \bar{e}_{i} e^{-i \omega_{i} t+i \phi_{i}}, G_{m m^{\prime}}(t)=-(i / 2) \Theta(t) \sum_{i} \bar{e}_{i m} \bar{e}_{i m^{\prime}} e^{-i \omega_{i} t}$ is the Green's function of phonons, $\Theta(t)$ the Heaviside step-function, and

$$
F(t) \simeq\left\langle n\left|\hat{a}_{l}^{+} \hat{a}_{l}(t)\right| n\right\rangle .
$$

Correlation functions of this type are well known in the theory of optical transitions (see, e.g. 10 ): $F(t)=n e^{-i \bar{\omega}_{l} t+f(t)}$, where $f(t)=-\Gamma t+\sum_{i}\left(V_{l l i}^{2} / 2 \hbar \omega_{i}^{3}\right)\left(e^{i \omega_{i} t}-1\right)$; the sum accounts for phonon transitions caused by a shift in their equilibrium positions in connection with the $n \rightarrow n-1$ transition in the mode, $\Gamma=-\dot{E}_{l} / \hbar \bar{\omega}_{l}$ is the decay constant, which should be determined self-consistently (if the relaxation is slow as compared to characteristic rates of the phonon Green's functions, then one can neglect the term $\Gamma t$ in this expression); $\hbar \bar{\omega}_{l}=\hbar \omega_{l}-\sum_{i} V_{l l i}^{2} / 2 \omega_{i}^{2}$ is the renormalized level spacing of the local mode. Equation (5) can be easily solved by means of a half-axis Fourier transform. One gets

$$
D(\omega)=R(\omega) D_{0}(\omega) ; \quad R(\omega)=\left[I-G(\omega) v \bar{G}^{*}\left(\omega_{l}-\omega\right) v\right]^{-1} D_{0}(\omega)
$$

$\left(v=V_{l} \sqrt{n}\right)$. Here the functions $D_{0}(\omega), G(\omega)$ and $\bar{G}^{*}(\omega)$ are the half-axis Fourier transforms of the functions $D_{0}(t)$, $G(t)$ and $G^{*}(t) e^{f(t)}$.

Inserting Eqs. (4) - (7) into (1) and averaging over $\phi_{i}$ (leading to the replacement of $e^{i\left(\phi_{i}-\phi_{i^{\prime}}\right)}$ by $\delta_{i i^{\prime}}$ ), one gets the following formula for the damping rate of the $n$th level of the mode:

$$
\Gamma(n) \simeq \frac{1}{2 \pi} \int_{0}^{\infty} d \omega S p\left[R(\omega) v \rho(\omega) R^{*}(\omega) v \bar{\rho}\left(\bar{\omega}_{l}-\omega\right)\right] .
$$

Here $\rho(\omega)=\operatorname{Im} G(\omega)$ and $\bar{\rho}(\omega)=\operatorname{Im} \bar{G}(\omega)$ are the usual and renormalized phonon density functions, respectively. The damping rate of the energy is $\dot{E}_{l, n}=\hbar \bar{\omega}_{l n} \Gamma(n)$ A comparison of this result with an analogous formula, obtained

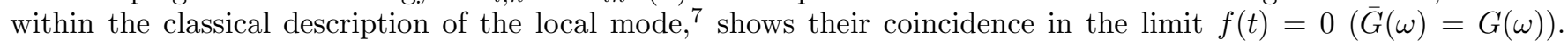
Such a difference should be expected: the function $f(t)$ accounts for phonon transitions, assisting the destruction of a quantum of the local mode, thus being of quantum mechanical origin.

For moderately large $n, \Gamma(n) \sim v(n)^{-1}$, i.e. the relaxation accelerates with time, which is analogous to $-\dot{E}_{l} \sim A^{-2}$ obtained in the classical description of the local moded. The acceleration continues down to the level $n_{c r}$, when the resolvent in $R(\omega)$ approaches zero (for $\omega \approx \bar{\omega}_{l} / 2$ and in some cases for other $\omega$ ). One can show that near such a level, $\Gamma(n) \sim\left|n-n_{c r}\right|^{-1} \sim\left|t-t_{c r}\right|^{-1 / 2}$, i.e. a sharp relaxation jump takes place accompanied by the generation of a burst of phonons (time $t_{c r}$ corresponds to $n=n_{c r}$ ). After a first jump, several other jumps may occur. For small $n$, formula (8) coincides with the result given by Fermi's Golden Rule (i.e. the standard perturbation theory). In the classical limit $\hbar \rightarrow 0$, the decay of the mode energy vanishes for phonons in the ground state.

The renormalized phonon density function $\bar{\rho}(\omega)$ is nonzero also for $\omega$ larger than the maximal phonon frequency $\omega_{M}$ (due to phonon-assisted transitions described by the function $f(t)$ ), while $\rho(\omega)$ is nonzero only in the one-phonon region $0<\omega<\omega_{M}$. Therefore formula (8) accounts not only for two-phonon decay ff the local mode but also for its three- and more-phonon decay (the corresponding formula for a classical local moded accounts for two-phonon decay only). The linear and quadratic interactions considered above are commonly present, enhancing or weakening each other, as follows from the value of the resolvent $R(\omega)$ in (8). As a result, three- and more-phonon processes may also cause relaxation jumps, e.g. if $\omega_{l} \geq 2 \omega_{M}$ and two-phonon processes are forbidden by the energy conservation law. If $\omega_{l} \gg 2 \omega_{M}$, the consideration of linear and quadratic terms with respect to phonon operators in the interaction hamiltonian is not sufficient; one needs to include higher order terms (see, in this connection, ref.E, where these terms have been considered within the classical description of the local mode). 
To illustrate the effect of three- and more-phonon processes according to the quantum formula (8), we performed a model calculation of the relaxatipm rates of a $\mathrm{Xe}_{2}^{*}$-molecule in solid Xe. Note that the relaxation of this system was recently studied experimentally in 11 where the relaxation jump was found to take place near the level $n=22$. The level spacing in a Xe-molecule is equal to $\hbar \omega_{M}(68-n) / 24$. Two-phonon relaxation is allowed for upper levels until $n=20$. Lower levels can decay with emission of no less than three phonons (their spacing exceeds $2 \hbar \omega_{M}$ ). Only the symmetrized displacement of two nearest atoms along the $\mathrm{Xe}_{2}^{*}$-molecule contributes significantly to the anharmonic interactions of this molecule with the environment (in this approximation, all matrices in (8) are scalars); the main contribution to this displacement comes from the longitudinal phonon 12. The corresponding density function is well approximated by the cap-type shape $\rho(x)=8 x \sqrt{1-x^{2}}, x=\omega / \omega_{M} 13$. Assuming $f(t)=-\Gamma t+\beta(G(t)-G(0))$, we calculated the $\Gamma(n)$-function for different values of the parameter $\beta \sim V_{l l m}$ (determining the shifts in the equilibrium positions of the phonon coordinates with the $n \rightarrow n-1$ transition of the local mode) and with the maximum at $n=22$. The results for $\gamma(n)=\Gamma(n) \omega_{l n} / \omega_{M}$ are presented in Fig.1. One sees that not only the two-phonondecay but also the three phonon decay is strongly enhanced in the vicinity of the critical level $n=22$. An estimation 11 gives for $\mathrm{Xe}_{2}^{*}$-molecule in $\mathrm{Xe}$ crystal $\beta \approx 0.2$.

Above the case $T=0$ was considered. Finite temperature effects are straightforward to take into account: in Eq. (8), there would appear an additional factor $1+2 n(\omega)$ under the integral and the factor $\left(e^{i \omega_{i} t}-1\right)$ in $f(t)$ should be replaced by the temperature-dependent factor $(n(\omega)+1)\left(e^{i \omega_{i} t}-1\right)+n(\omega)\left(e^{-i \omega_{i} t}-1\right)$ (here $\left.n(\omega)=1 /\left(e^{\hbar \omega / k T}-1\right)\right)$. The formulae obtained are applicable generally for nonadiabatic transitions in the case of linear diagonal and quadratic nondiagonal interactions of the system with the phonon continuum.

In conclusion, we have presented a nonperturbative theory of multiphonon anharmonic quantum transitions between levels of a local mode taking into account linear and quadratic terms with respect to phonon operators. We showed that for high levels, transitions accelerate in time which is in contradiction with the standard perturbation theory. This continues down to the level $n_{c r}$, when the rate of the process becomes large - of the order of $\omega_{M}^{-1}$, and a sharp relaxation jump takes place, accompanied by the generation of a burst of phonons. Depending on the level spacing, phonons may be emitted in pairs or triplets, quartets and etc. After the first jump, several other jumps may occur. Only for low levels standard perturbation theory and Fermi's Golden Rule are applicable. This theory can be applied generally for the nonperturbative description of nonadiabatic transitions caused by linear diagonal and quadratic nondiagonal interactions of the system with the phonon continuum.

The author is grateful to Dr. M. Selg for his assistance concerning the enhancement of the three-phonon relaxation. This research was supported by Estonian Science Foundation, Grant No. 2274 and by the DAAD Grant HSPS.

${ }^{1}$ Ovchinnikov A.A., Zh. Eksp. Teor. Fiz. 57 (1969) 263. [Sov. Phys. JETP 30 (1970) 147]; Ovchinnikov A.A.and Erihman N.S., Usp. Fiz. Nauk 138 (1982) 290. [Sov. Phys. Usp. 25 (1982) 738].

${ }^{2}$ Kosevich A.M.,Kovalev A.S., Zh. Eksp. Teor. Fiz. 67 (1974) 1793 [Sov. Phys. JETP, 40 (1974) 907].

${ }^{3}$ Dolgov A.A., Fiz. Tverd. Tela (Leningrad) 28 (1986) 164. [Sov. Phys. Solid State 28 (1986) 907.

${ }^{4}$ Sievers A.J., Takeno S., Phys. Rev. Lett. 61 (1988) 970.

${ }^{5}$ Flach S., Willis C.R., Physics Report 295 (1998) 181.

${ }^{6}$ Klemens P., Phys. Rev.122 (1961) 443.

${ }^{7}$ Hizhnyakov V., Phys.Rev. B53 (1996) 13981; Proceedings of the XIIth Symposium on the Jahn-Teller Effect, Tartu 1994 [Proc. Estonian Acad. Sci. Phys. Math. 44 (1995) 364]; Hizhnyakov V., Nevedrov D., Phys. Rev. B 56 (1997) R2908.

8 Hizhnyakov V., Z.Phys. B 104 (1997) 675.

${ }^{9}$ Klinger M.I., Sov. Phys. -Usp. 30 (1987) 699.

${ }^{10}$ Lax M., Chem. Phys. 20 (1952) 1752.

${ }^{11}$ Hizhnyakov V., Selg M., Kink M., Kink R., and Maksimov J., J.Low Temp. Phys. 111 (1998) 709.

12 Anselm A.I., Firsov Yu.A., JETP 28 (1955) 151; 30 (1956) 719.

${ }^{13}$ Lurie N.A., Shirane G., Skalyo J., Phys. Rev. B 9 (1974) 5300. 


\section{Figure caption}

Fig. 1. Theoretical relaxation rate $\gamma\left(\right.$ in $\omega_{M} \approx 8.3 \mathrm{sec}^{-1}$ units) vs level number $n$ of a Xe $\mathrm{X}_{2}^{*}$-molecule in solid Xe for different values of the phonon displacement parameter $\beta$. 

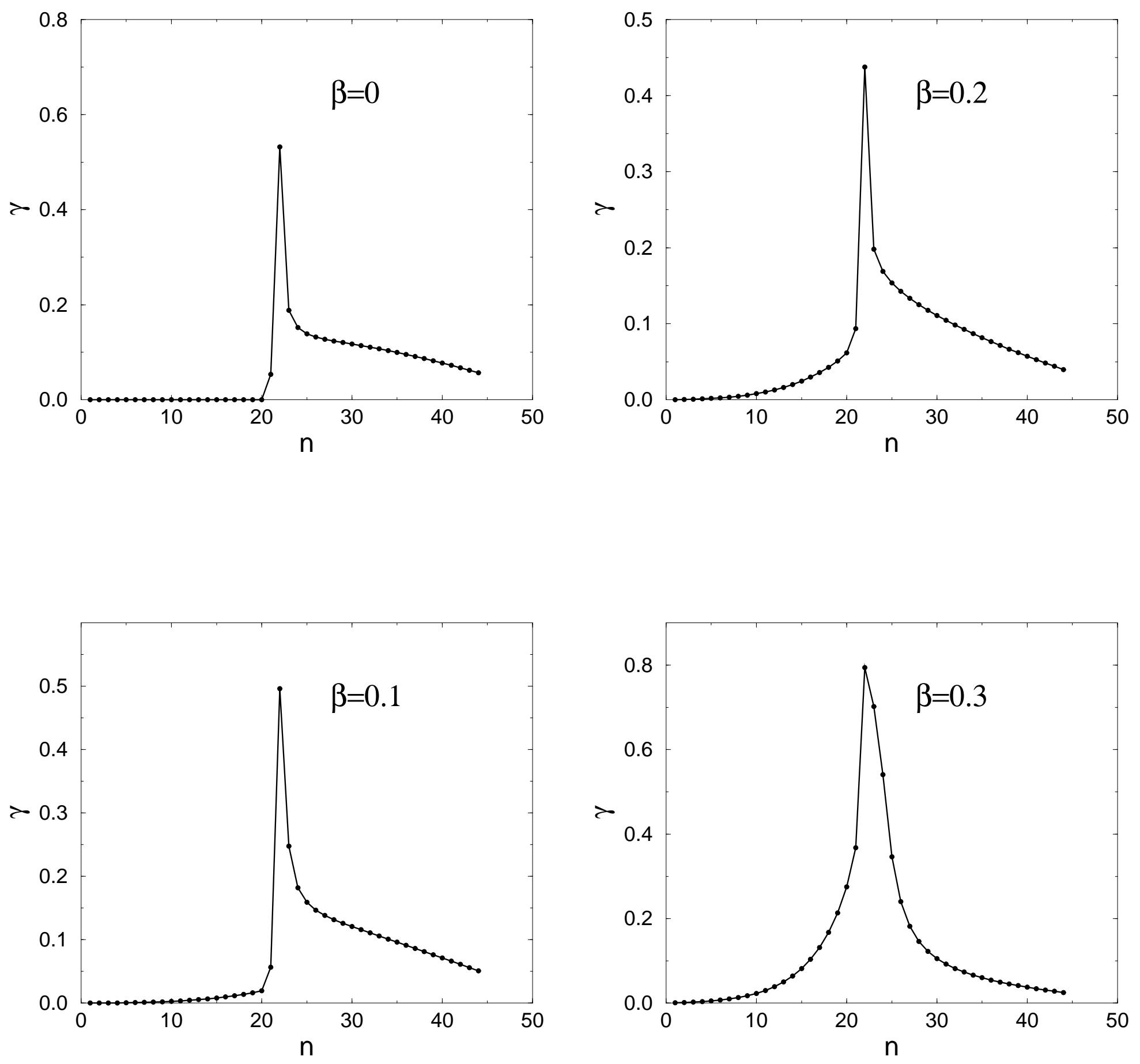